\title{
TU3B-(2)-4
}

\section{RATE-EQUATION MODELING OF SPECTRUM AND DYNAMIC PROPERTIES OF QUANTUM-DOT LASERS}

\author{
H.-T. Chen, W.-H. Hsieh, and M.-H. Mao \\ Department of Electrical Engineering, Graduate Institute of Electronics Engineering, and \\ Graduate Institute of Electro-Optical Engineering, National Taiwan University \\ No.1, Sec. 4, Roosevelt Road, Taipei, Taiwan 106, R.O.C. \\ Phone: 02-23635251, FAX: 02-23681679, E-mail: mhmao@cc.ee.ntu.edu.tw
}

\begin{abstract}
The influences of the material and structural parameters on the static and dynamic characteristics of quantum-dot lasers are analyzed using a rate equation model. The simulation results are also compared with those of quantum-well lasers.
\end{abstract}

\section{INTRODUCTION}

Quantum-dot (QD) lasers have been demonstrated to have excellent static characteristics [1]. However, further theoretical investigations on their dynamic properties are still needed. In this paper, we use a rate equation model proposed in $[2,3]$ to simulate the static and dynamic characteristics of QD lasers. The influences of the material and structural parameters, such as homogeneous and inhomogeneous broadening, and various time constants, on the static and dynamic characteristics of quantum-dot lasers are analyzed. We also compare the simulation results of QD lasers with those of quantum-well (QW) lasers.

\section{SIMULATION RESULTS}

The transient behaviors and energy spectra of QD lasers are simulated using rate equations with an appropriate optical gain model [2]. The lasing spectrum of QD lasers demonstrates a sharp peak at $300 \mathrm{~K}$ (homogeneous broadening $20 \mathrm{meV}$ ), but it will be broadened at lower temperatures (homogeneous broadening $0.2 \mathrm{meV}$ ), the same as that indicated in [2]. This provides a verification of the correctness of our simulations. Concerning the dynamic properties, the relaxation oscillations of QD lasers are largely damped with carrier

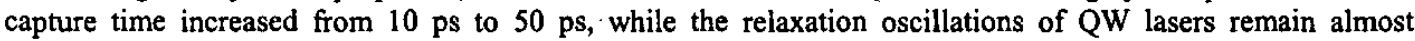
unchanged. It is indicated that the "phonon bottleneck" effect [4] will cause a considerable carrier capture time into QDs. Through the simulation above, the effect of a large carrier capture time on QD lasers is shown to be much more pronounced than that on QW lasers. On the other hand, the relaxation oscillation frequency of QD lasers decreases with increasing inhomogeneous broadening. Therefore, the inhomogeneous broadening due to the size fluctuation of QDs needs to be reduced in order to achieve faster relaxation oscillations and therefore higher modulation bandwidth.

\section{ACKNOWLEDGMENTS}

This work was partially supported by the National Science Council, Republic of China, under the Grant No. NSC 91-2120-E-002-006.

\section{REFERENCES}

[1] D. Bimberg, M. Grundamann, and N. N. Ledentsov, Quantum Dot Heterostructures, John Wiley and Sons (1999).

[2] M. Sugawara, K. Mukai, Y. Nakata, and H. Ishikawa, "Effect of homogeneous broadening of optical gain on

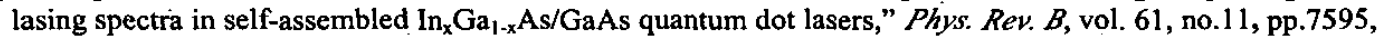
March (2000).

[3] M. Sugawara, Self-Assembled InGaAs/GaAs Quantum Dots, Academic press (1999).

[4] H. Benisty, C. M. Sotomayor-Torres, and C. Weisbuch, "Intrinsic mechanism for the poor luminescence properties of quantum-box systems," Phys. Rev. B, vol. 44, pp. 10945 (1991). 